\title{
Title: Rhoptry neck protein RON2 forms a complex with microneme protein AMA1 in
}

\section{Plasmodium falciparum merozoites}

\author{
Jun Cao ${ }^{\mathrm{a}, \mathrm{b}}$, Osamu Kaneko, ${ }^{\mathrm{a}, \mathrm{c},}$, Amporn Thongkukiatkul ${ }^{\mathrm{d}}$, Mayumi Tachibana ${ }^{\mathrm{a}}$, \\ Hitoshi Otsuki $i^{\mathrm{a}}$, Qi Gao ${ }^{\mathrm{b}}$, Takafumi Tsuboi ${ }^{\mathrm{e}, \mathrm{f}}$, Motomi Torii ${ }^{\mathrm{a}}$
}

${ }^{a}$ Department of Molecular Parasitology, Ehime University Graduate School of Medicine, Shitsukawa, Toon, Ehime 791-0295, Japan

${ }^{b}$ Malaria Department, Jiangsu Institute of Parasitic Diseases, Meiyuan, Wuxi, Jiangsu 214064, People's Republic of China

${ }^{c}$ Department of Protozoology, Institute of Tropical Medicine (NEKKEN), Nagasaki University, Sakamoto, Nagasaki 852-8523, Japan

${ }^{d}$ Department of Biology, Faculty of Science, Burapha University, Chonburi 20131, Thailand

${ }^{e}$ Cell-Free Science and Technology Research Center, Ehime University, Matsuyama, Ehime 790-8577, Japan

${ }^{f}$ Venture Business Laboratory, Ehime University, Matsuyama, Ehime 790-8577, Japan

Abbreviations: aa, amino acid(s); Ab, antibody; AMA1, apical membrane antigen 1; GST, Glutathione S transferase; PBS, phosphate-buffered saline; PCR, polymerase chain reaction; RON, rhoptry neck protein.

* Corresponding author: Tel.: (+81) 95819 7838; Fax: (+81) 95819 7805; E-mail address: okaneko@nagasaki-u.ac.jp 
Sequence data from this article have been deposited with the GenBank ${ }^{\mathrm{TM}} / \mathrm{EMBL} / \mathrm{DDBJ}$ databases under accession numbers AB444588-AB444592.

\section{Abstract}

Erythrocyte invasion is an essential step in the establishment of host infection by malaria parasites, and is a major target of intervention strategies that attempt to control the disease. Recent proteome analysis of the closely-related apicomplexan parasite, Toxoplasma gondii, revealed a panel of novel proteins (RONs) located at the neck portion of the rhoptries. Three of these proteins, RON2, RON4, and RON5 have been shown to form a complex with the microneme protein Apical Membrane Protein 1 (AMA1). This complex, termed the Moving Junction complex, localizes at the interface of the parasite and the host cell during the invasion process. Here we characterized a RON2 ortholog in Plasmodium falciparum. PfRON2 transcription peaked at the mature schizont stage and was expressed at the neck portion of the rhoptry in the merozoite. Co-immunoprecipitation of PfRON2, PfRON4 and PfAMA1 indicated that the complex formation is conserved between $T$. gondii and $P$. falciparum, suggesting that co-operative function of the rhoptry and microneme proteins is a common mechanism in apicomplexan parasites during host cell invasion. PfRON2 possesses a region displaying homology with the rhoptry body protein $P f R h o p H 1 / C l a g$, a component of the RhopH complex. However, here we present co-immunoprecipitation studies which suggest that PfRON2 is not a component of the RhopH complex and has an independent role. Nucleotide polymorphism analysis suggested that PfRON2 was under diversifying selective pressure. This evidence suggests that RON2 appears to have a fundamental role in host cell invasion by apicomplexan parasites, and is a potential target for malaria intervention strategies. 
Keywords: AMA1; erythrocyte invasion; merozoite; Plasmodium falciparum; rhoptry

\section{Introduction}

Malaria is one of the most prevalent and deadly global infectious diseases, more than half of the world's population is at the risk of infection, and over 300 million people develop clinical disease each year of which 2 million are fatal [1]. Clinical malaria results from the replication of protozoan parasites of the genus Plasmodium in the circulating erythrocytes of the host. During the time between release from a rupturing mature schizont-infected erythrocyte and invasion of new erythrocytes, merozoites are transiently exposed in the circulation, and are thus potentially vulnerable to attack by preventive measures based upon immunological or biochemical methods. To design such tools, it is important to understand the molecular composition of the merozoite and the structure-function makeup of the molecular interactions that occur as the merozoite recognizes and gains entry into a host cell.

Like most apicomplexan parasites, the malaria merozoite invades host cells via a multistep process initiated by reversible binding to the erythrocyte surface. Subsequently, a high affinity attachment occurs between the apical end of the merozoite and the host cell, followed by the movement of the junctional adhesion zone (moving junction) around the merozoite toward its posterior pole. Finally the merozoite invaginates into the erythrocyte by forming a nascent parasitophorous vacuole [2]. The moving junction is one of the most distinctive features of apicomplexan invasion and was first observed in Plasmodium species in the late 1970s [3], but the molecular nature of its structure remains unresolved.

Recent studies in Toxoplasma gondii suggest that host cell invasion involves protein 
discharge from at least two apical secretory organelles, the micronemes and rhoptries, based on the observation that a microneme protein, Apical Membrane Protein 1 (AMA1), forms a complex with three rhoptry neck (RON) proteins: RON2, RON4 and Ts4705 (RON5) [4 - 6]. These proteins have predicted orthologs in P. falciparum, and the RON4 ortholog has been reported to associate with PfAMA1 [7] and to be localized at the moving junction [8], suggesting that the complex (and likely its function) is conserved between $T$. gondii and $P$. falciparum [7]. Attempts to knock-out the AMA1 gene locus were unsuccessful in both Plasmodium [9] and T. gondii [10], and the conditional reduction of TgAMA1 expression severely impaired the cell invasion ability of T. gondii [11], indicating AMA1 has an essential function. The conservation of the RON proteins among apicomplexan parasites suggest that their functions and protein interactions are also conserved in the biology of host cell invasion. However, in Plasmodium, the details of this complex have yet to be fully characterized. In this study, to better understand the moving junction complex formation in Plasmodium, we sought

to characterize PfRON2 and determine the nature of its interaction with PfRON4 and PfAMA1.

\section{Materials and methods}

\subsection{Malaria parasites}

P. falciparum cloned lines 3D7, HB3, Dd2, 7G8, FVO, and D10 were maintained in vitro, essentially as previously described [12].

\subsection{DNA and RNA isolation}


Genomic DNA (gDNA) was isolated from $P$. falciparum using IsoQuick ${ }^{\mathrm{TM}}$ (Orca Research Inc., Bothell, WA). To determine transcription levels throughout the asexual stages, schizonts were purified by differential centrifugation on a $70 \% / 40 \%$ Percoll-sorbitol gradient, after which released merozoites were allowed to invade uninfected erythrocytes for 4 hours before the clearance of all remaining schizonts using 5\% D-sorbitol. Fractions of the culture were harvested immediately and 24 hours later, and then at 6 hour intervals thereafter. Total RNA was isolated from parasite-infected erythrocytes stored at $-20^{\circ} \mathrm{C}$ in RNAlater ${ }^{\mathrm{TM}}$ (Qiagen, Valencia, CA), using the RNeasy Mini Kit (Qiagen). Following DNase treatment, complementary DNA (cDNA) was generated with random hexamers using an Omniscript Reverse Transcription Kit (Qiagen).

\subsection{Polymerase chain reaction (PCR) amplification and sequencing}

A TBLASTN search was performed against the $P$. falciparum genome database (3D7 parasite line) via PlasmoDB website (http://www.plasmodb.org/) [13] using the TgRON2 amino acid sequence as a query. To evaluate the polymorphism of $P f R O N 2$, five pairs of overlapping primers were used for PCR amplification from HB3, FVO, Dd2, D10, and 7G8 parasite lines, and sequences were determined by direct sequencing of the PCR-amplified DNA fragments using an ABI PRISM ${ }^{\circledR}$ 3100-Avant Genetic Analyzer (Applied Biosystems, Foster City, CA). Oligonucleotides used were as follows: fRON2.F2 (5’-GATTCCAATAATTATAATTCTGATAATG-3') fRON2.R2

(5'-CGTAAAATATTCATTATATGAAAGATATGC-3’), fRON2.F3

(5'-GCATTAGGAGAACTTGTTGAACCA-3') and fRON2.R3

(5'-CATAATATCTAAATAGGTTTTTGCTGAC-3'), fRON2.F4 (5'-GGATTAGTATTTTTATATGCAATGATTG-3') and fRON2.R4 
(5'-GTTATTTTCTAATAAATGTTTACTATCTTC-3'), fRON2.F5

(5'-GATAAATGGGATCAATTTATAAATAAGG-3') and fRON2.R5 (5'-GCTAGCTACTGGTCCTGCACCT-3'), and fRON2.F6 (5'-ATGCAATTACCTTACTTAAGTCAAATG-3') and fRON2.R6 (5'-ATATAAAATGAAAATAACAGAAAAGGTTATG-3')

\subsection{Quantification of pfron2 transcripts}

Transcription of ron2 was evaluated in the HB3 parasite line by real-time reverse transcription (RT)-PCR using a QuantiTect SYBR Green PCR Kit (Qiagen) and a LightCycler System (Roche, Basel, Switzerland). As a control, transcription of ama1 and rhoph2 was also evaluated. Oligonucleotides used were as follows: fRON2.qF (5'-CAGAACTAAGCAAACATGTAAAACATG-3') and fRON2.qR (5'-GTATAACGCCTTGCTCATTTCCTG-3') for pfron2 (product size is 133 bp); fAMA1.qF (5'-GGAAGAGGACAGAATTATTGGGAAC-3') and fAMA1.qR (5'-CCTGAATCTTCTTGTTGGTATGTATG-3') for pfama1 (product size is $137 \mathrm{bp}$ ); fRhopH2.qF (5'-GTAACAACACTTACTAAGGCAGACT-3') and fRhopH2.qR (5'-GTACAAAGCTACAATATTGTTAGATCT-3') for pfrhoph2 (product size is $210 \mathrm{bp}$ ). The same oligonucleotides were used to PCR-amplify DNA fragments to be ligated into the pGEM-T Easy ${ }^{\circledR}$ plasmid (Promega, Madison, WI) which was used to make a standard curve to evaluate the copy number of each transcript.

\subsection{Antibodies}


A DNA fragment encoding amino acid positions (aa) $21-98$ of PfRON2 was PCR-amplified from $P$. falciparum 3D7 gDNA and ligated into pEU-E01GST-N2, an expression plasmid with N-terminal glutathione S transferase (GST)-tag followed by a PreScission Protease cleavage site, designed specifically for the wheat germ cell-free protein expression system (CellFree Sciences Co., Ltd., Matsuyama, Japan) [14], to produce recombinant GST-fused fRON2N protein (GST-fRON2N). Oligonucleotides used in the PCR amplification were fRON2.SalF1 (5'-GTCGACTCAGAACTAAGCAAACATGTAAAACATG-3') and fRON2.SalR1 (5'-GTCGACCCCATTATTCATTTCACTACCAGGA-3') (SalI restriction sites are underlined). Produced GST-fRON2N was captured using a glutathione-Sepharose 4B column and eluted with $10 \mathrm{mM}$ reduced gluthathione, $\mathrm{pH} 8.0$. To generate anti-PfRON2 sera, BALB/c mice were immunized subcutaneously with $20 \mu \mathrm{g}$ of purified GST-fRON2N emulsified with Freund's adjuvant. A Japanese white rabbit was immunized subcutaneously with $500 \mu \mathrm{g}$ of purified GST-fRON2N with Freund's adjuvant for the first time, followed by $250 \mu \mathrm{g}$ thereafter. All immunizations were done 4 times at 3 week intervals, prior to collection of antisera. Rabbit anti-PfRhopH2 serum was obtained from I. Ling (National Institute for Medical Research, UK) [15], Rabbit anti-PfAMA1 serum was obtained from C. Long (National Institute of Health, USA), and mouse monoclonal anti-PfRON4 antibody (Ab; 26C64F12) was obtained from J.-F. Dubremetz (Université de Montpellier 2, France) [7]. Rabbit anti-Clag3.1 serum was as previously described [16].

\subsection{SDS-PAGE and Western blot analysis}

The recombinant protein, GST-fRON2N, was digested with a PreScission Protease at $4{ }^{\circ} \mathrm{C}$ overnight before analysis. Triton X-100 extracts of $P$. falciparum or recombinant proteins 
were dissolved in SDS-PAGE loading buffer, incubated at $100^{\circ} \mathrm{C}$ for $3 \mathrm{~min}$, and subjected to electrophoresis under reducing conditions on a 5-20\% polyacrylamide gel (ATTO, Japan). Proteins were then transferred to a $0.22 \mu \mathrm{m}$ PVDF membrane (BioRad, Hercules, CA). The proteins were immunostained with antisera followed by horseradish peroxidase-conjugated secondary $\mathrm{Ab}$ (Biosource Int., Camarillo, CA) and visualized with Immobilon ${ }^{\mathrm{TM}}$ Western Chemiluminescent HRP Substrate (Millipore, Billerica, MA) on RX-U film (Fuji, Japan). The relative molecular sizes of the parasite-encoded proteins were calculated by reference to molecular size standards (BioRad).

\subsection{Immunoprecipitation}

Immunoprecipitation was carried out as previously described [17]. Briefly, proteins were extracted from late schizont parasite pellets by $1 \%$ Triton $\mathrm{X}-100$ treatment in phosphate-buffered saline (PBS) containing cOmplete Proteinase Inhibitor Cocktail Tablets (Roche). Supernatants $(50 \mu \mathrm{l})$ were pre-incubated at $4^{\circ} \mathrm{C}$ for 1 hour with $20 \mu \mathrm{l}$ of $50 \%$ protein G-conjugated beads (GammaBind Plus Sepharose; GE Healthcare) in NETT buffer (50 mM Tris- $\mathrm{HCl}, 0.15 \mathrm{M} \mathrm{NaCl}, 1 \mathrm{mM}$ EDTA, and $0.5 \%$ Triton X-100) supplemented with $0.5 \%$ BSA (fraction V; Sigma-Aldrich). Recovered supernatants were incubated with rabbit antisera (anti-PfRON2, anti-PfAMA1, or anti-PfRhopH2) or mouse anti-PfRON4 Ab with gentle rotation at $4{ }^{\circ} \mathrm{C}$ for 2 hours and then $20 \mu \mathrm{l}$ of $50 \%$ protein G-conjugated beads were added. After 1 hour incubation at $4^{\circ} \mathrm{C}$, the beads were washed once with NETT- $0.5 \%$ BSA, once with NETT, once with high-salt NETT $(0.5 \mathrm{M} \mathrm{NaCl})$, once with NETT, and once with low-salt NETT (0.05 M NaCl and $0.17 \%$ Triton X-100). Finally, proteins were extracted from the protein G-conjugated beads by incubation with SDS-PAGE reducing loading buffer at $100^{\circ} \mathrm{C}$ for $3 \mathrm{~min}$. Supernatants were collected for Western blot analysis. 


\subsection{Indirect immunofluorescence assay}

Thin smears of schizont-enriched P. falciparum-infected erythrocytes (Dd2 parasite line) were prepared on glass slides and stored at $-80^{\circ} \mathrm{C}$. The smears were thawed, formaldehyde-fixed, and preincubated with PBS containing $5 \%$ non-fat milk at $37^{\circ} \mathrm{C}$ for 30 min. They were then incubated with antisera at $37^{\circ} \mathrm{C}$ for 1 hour, followed by fluorescein isothiocyanate (FITC)-conjugated goat anti-(IgG and $\operatorname{IgM})$ secondary $\mathrm{Ab}$ (Jackson ImmunoResearch Laboratories, West Grove, PA) and Alexa546-conjugated goat anti-(IgG and $\mathrm{IgM}$ ) secondary $\mathrm{Ab}$ (Invitrogen, Carlsbad, CA) at $37^{\circ} \mathrm{C}$ for $30 \mathrm{~min}$. Nuclei were stained with 4',6-diamidino-2-phenylindole (DAPI). Slides were mounted in ProLong Gold antifade reagent (Invitrogen) and viewed under oil-immersion. High resolution image-capture and processing were performed using a confocal scanning laser microscope (LSM5 PASCAL; Carl Zeiss MicroImaging, Thornwood, NY). Images were processed in Adobe Photoshop (Adobe Systems Inc., San José, CA).

\subsection{Immunoelectron microscopy}

Parasites were fixed for $15 \mathrm{~min}$ on ice in a mixture of $1 \%$ paraformaldehyde- $0.1 \%$ glutaraldehyde in $0.1 \mathrm{M}$ phosphate buffer ( $\mathrm{pH}$ 7.4). Fixed specimens were washed, dehydrated, and embedded in LR White resin (Polysciences, Inc., Warrington, PA) as previously described $[18,19]$. Thin sections were blocked at $37^{\circ} \mathrm{C}$ for $30 \mathrm{~min}$ in $\mathrm{PBS}$ containing 5\% non-fat milk and 0.01\% Tween 20 (PBS-MT). Grids were then incubated at $4^{\circ} \mathrm{C}$ overnight with mouse anti-PfRON2 or control sera in PBS-MT. After washing with PBS containing 10\% BlockAce (Yukijirushi, Sapporo, Japan) and $0.01 \%$ Tween 20 (PBS-BT), the 
grids were incubated at $37^{\circ} \mathrm{C}$ for 1 hour with goat anti-mouse IgG conjugated to $10 \mathrm{~nm}$ gold particles (Amersham Life Science, Arlington, IL) diluted 1:20 in PBS-MT, rinsed with PBS-BT, and fixed on ice for $10 \mathrm{~min}$ in $2.5 \%$ glutaraldehyde to stabilize the gold. Then the grids were rinsed with distilled water, dried, and stained with uranyl acetate and lead citrate. Samples were examined with a transmission electron microscope (JEM-1230; JEOL Ltd., Tokyo, Japan).

\subsection{Primary structure analysis of the protein}

Signal peptide sequence was evaluated by SignalP3.0 [20]. Transmembrane region was evaluated by TMpred [21] and TMHMM2.0 [22]. Low complexity region was evaluated by Globplot 2.3 [23]. Amino acid sequence alignment was generated by MUSCLE [24].

\subsection{Statistical analysis}

Number of nonsynonymous substitutions over numbers of nonsynonymous sites $\left(d_{N}\right)$, number of synonymous substitutions over numbers of synonymous sites $\left(d_{\mathrm{S}}\right)$, and their standard errors were computed using the Nei-Gojobori method with Jukes-Cantor correction implemented in MEGA 4.0.1 [25]. Standard errors were estimated using the bootstrap method with 500 replications. The statistical difference between $d_{\mathrm{N}}$ and $d_{\mathrm{S}}$ was tested using a one-tail Z-test with 500 bootstrap pseudosamples.

\section{Results}

\subsection{RON2 orthologs of apicomplexan parasites}


Using TgRON2 as a query in BLAST analyses [26], and similar analyses using the predicted orthologs thus identified, we found RON2 orthologs in P. falciparum (PfRON2; PF14_0495, PlasmoDB), P. yoelii 17XNL strain (PyRON2; PY06813, TIGR), P. knowlesi H strain (PkRON2; PKH_125430 or PK14_2335w, Sanger Centre), and P. vivax Sal-I strain (PvRON2; Pv117880, TIGR), P. berghei (PbRON2; Contig5108), P. chabaudi (PchRON2; Contig882.0), Theileria annulata (TaRON2; Fig. S1A, TA19445 and TA19390, Sanger Centre [27]), Theileria parva (TpRON2; Fig. S1B, TP01_0014, TIGR [28]), and Babesia bigemina (BbigRON2; Fig. S1C, Contig3449, Sanger Centre). The RON2 were fragmented in the P. berghei, P. chabaudi, T. annulata, and T. parva genome nucleotide sequence databases, and full-length versions were constructed (supplementary Table S1).

\subsection{PfRON2 protein structure and similarity to RhopH1/Clag proteins}

The full-length PfRON2 protein consists of 2189 residues with a putative signal peptide sequence at its $\mathrm{N}$-terminus from amino acid positions (aa) 1 to 20 . An interspecies variable region (aa 55 - 878), exhibiting low complexity and many repeats [23], was identified by comparing 6 Plasmodium RON2 amino acid sequences (Fig. 1 and Fig. S2). A BLASTP search using the conserved region of PfRON2 (aa $879-2189$ ) as a query identified P. vivax RhopH1/Clag homolog (XP_001616939.1, aa 251 - 394; E = 0.001) as possessing homology with PfRON2 aa 1105 - 1259. A Position-Specific Iterated BLAST search using PfRON2 aa $1105-1259$ as a query converged at iteration 3 and indentified most of the RhopH1/Clag genes in Plasmodium species. Alignment of RhopH1/Clag with RON2 from multiple genera identifies a predicted globular domain that is likely stabilized by disulfide bonds between 4 conserved Cys residues (Fig. 2). Three transmembrane regions were predicted by TMpred, 
however TMHMM2.0 predicted only a single transmembrane region for all Plasmodium RON2 orthologs assessed.. Interestingly, TMpred predicted a putative transmembrane region in the region conserved between RhopH1/Clag and RON2 (Fig. 2). Because RhopH1/Clag is a component of a soluble protein complex, we considered that these predicted transmembrane regions in RhopH1/Clag and RON2 constitute a likely hydrophobic region buried within a globular domain. Another predicted transmembrane region at aa $1114-1133$ in PfRON2 is also possibly hydrophobic region buried within a globular domain. TMpred considers the observation that there is an overrepresentation of positively charged amino acid residues in the cytoplasmic loops of the transmembrane protein [21], which is a likely explanation for this discrepancy.

\subsection{PfRON2 transcription peaks at the schizont stage}

To determine the transcription pattern in the asexual stages of the parasite life-cycle, quantitative RT-PCR was performed on the HB3 parasite line prepared from a synchronized culture harvested at 6 hour intervals. Both RON2 and AMA1 transcriptions were seen to peak around $36-40$ hours after invasion, when parasites were in the schizont stage. AMA1 showed a broader and flatter transcription peak than RON2 (Fig. 3). Transcriptome data compiled in the PlasmoDB website $[13,29]$ also indicated a milder wave crest of AMA1 transcripts compared with RON2.

\subsection{Complex formation of PfRON2, PfRON4, and PfAMA1}

Mouse and rabbit anti-PfRON2 sera were generated using recombinant GST-fRON2N. Firstly, we evaluated the reactivity of anti-PfRON2 sera by Western blot using recombinant 
proteins. Both antisera recognized the fRON2N component of the recombinant protein after cleavage (Fig. S3, filled arrows). Cleaved 26.4-kDa GST component (Fig. S3, arrowheads) and 46-kDa GST-fused PreScission protease (Fig. S3, unfilled arrow) were also recognized by these Abs.

Secondly, we evaluated the reactivity of these sera against native RON2 proteins extracted from schizont stage $P$. falciparum (HB3 line) by Western blot analysis. Both antisera reacted with a band slightly larger than $250 \mathrm{kDa}$ (Fig. 4A, arrows), which is similar to the predicted molecular weight of PfRON2 after exclusion of the putative signal peptide sequence $(247 \mathrm{kDa})$. An $80-\mathrm{kDa}$ band was detected by both mouse and rabbit antisera in HB3 extract, for which the exact identity is not known, but a possible processed product of PfRON2. A 55-kDa band detected with rabbit antiserum was also detected with preimmune serum, suggesting that this band was unrelated to PfRON2. A 35-kDa band was detected with mouse antiserum but not with rabbit antiserum, suggesting that it is also unrelated to RON2.

To evaluate the interaction between PfRON2, PfRON4, and PfAMA1, we performed immunoblotting against immunoprecipitated materials from mature schizont-rich parasite extracts (Fig. 5). We found that RON2 was detected in the precipitated fraction using anti-PfAMA1 or anti-PfRON4. In the reciprocal experiment, PfAMA1 and PfRON4 were also detected in the precipitated fraction of anti-PfRON2 serum. Although it is theoretically possible that such immunoprecipitated fractions contained the PfRON2-PfRON4, PfRON2-PfAMA1, and PfRON4-PfAMA1 dimeric complexes as appropriate to the primary antibody, considering that these 3 proteins are distinct molecules that do not possess any similarity each other, this specific co-immunoprecipitation suggests complex formation among PfRON2, PfRON4, and PfAMA1 in P. falciparum. The fact that both the $83-\mathrm{kDa}$ proform and the 66-kDa processed form were co-precipitated with PfRON2 indicated that a region responsible for complex formation was located in the 66-kDa form of AMA1 [30]. 
Neither of these was detected in the anti-RhopH2 immunoprecipitate, thereby excluding not only the possibility of PfRON2 involvement in the RhopH complex, but also potential carryover due to insufficient or inadequate washing steps.

\subsection{RON2 is expressed at the rhoptry neck of Plasmodium merozoites}

Dual labeling indirect immunofluorescent assay was performed using anti-PfRON2 with either anti-PfAMA1 (microneme marker), anti-Clag3.1 (rhoptry body marker), or anti-PfRON4 (rhoptry neck marker) antibodies in order to determine the sub-cellular location of PfRON2 in P. falciparum (Fig. 6). In segmented schizonts, RON2 antisera produced a punctate pattern of fluorescence and each developing merozoite showed a single small punctate PfRON2-positive signal located at the apical end. Although some parts of the PfRON2 signal overlapped with microneme protein AMA1 and rhoptry body protein Clag3.1, it did not colocalize well with those markers, whereas complete colocalization was observed with the rhoptry neck marker PfRON4.

Immunoelectron microscopy was carried out to determine the precise localization of the protein. PfRON2 was detected in the neck portion of the pear-shaped rhoptries in segmented schizonts (Fig. 7). Thus PfRON2 is seen to compartmentalize in the rhoptry neck.

\subsection{Potential positive diversifying selection on PfRON2}

To evaluate the polymorphic nature of PfRON2, we sequenced the pfron2 nucleotide sequence $(2459-6570)$, excluding the $5^{\prime}$ low complexity region, in 5 P. falciparum parasite lines and compared them with the sequence from the genome database (3D7 line). A total of 5 nonsynonymous nucleotide substitutions were observed at nucleotide positions 2615,2710 , 
2914, 4391 and 4392, resulting 4 amino acid substitutions (Table 1). An excess of nonsynonymous substitutions $\left(d_{\mathrm{N}}=0.0007 \pm 0.0003\right)$ over synonymous substitutions $\left(d_{\mathrm{S}}=\right.$ $0.0002 \pm 0.0002)$ was detected $(P=0.0333)$, indicating PfRON2 is subject to positive diversifying selection.

\section{Discussion}

In this study, we characterized $P$. falciparum RON2 for its protein structure, transcription profiles, intracellular localization, and complex formation with PfRON4 and PfAMA1.

PfRON2 possesses a region harboring homology with another rhoptry protein RhopH1/Clag, a component of the RhopH complex that possesses erythrocyte binding ability $[16,31,32]$. Co-immunoprecipitation showed that PfRON2 does not form a complex with RhopH2, suggesting that PfRON2 is unlikely to be a component of the RhopH complex. Because RON2 orthologs can be found in other apicomplexan parasites and RhopH1/Clag is found only in Plasmodium species, RhopH1/Clag probably evolved via acquisition of a conserved functional domain from RON2 during its generation in Plasmodium species. Thus, this homologous region may have a common function between these two complexes. The sequence of TgRON2 deposited to the database (GenBank accession number DQ096563) only

possesses the C-terminal half of the conserved region between RON2 and RhopH1/Clag. By comparing TgRON2 gDNA and cDNA sequences, we noticed that intron 3 is relatively large (2272 bp) and contains a potential sequence encoding the N-terminal portion of the conserved region. Thus it is possible that there is another alternatively spliced transcript encoding the full length of the conserved region. Alternatively, it is also possible that this region represents an ancient vestigial exon. 
Interestingly, we could readily detect complex formation between AMA1 and RON proteins in the extract obtained from mature schizont-rich parasites, suggesting that complex formation had already occurred at the schizont stage likely at the apical end upon secretion of RON proteins from rhoptry and AMA1 from microneme. This is in contrast to the other apicomplexa parasite $T$. gondii, in which the AMA1-RON complex was proposed to form at the initial contact with the host cell. The precise timing of the complex formation is not clear, but may vary depending on the parasite species. Among RON proteins characterized thus far, only TgRON4 was visualized to locate at the moving junction during cell invasion. Whether $P f R O N 2$ and $P f R O N 4$ locate at the moving junction and whether the complex remains intact during cell invasion are still need to be clarified. We found that PfRON2 degraded more rapidly than PfRON4 after extraction (Fig. S4), which may explain the previous observation by Alexander et al. (2006), who did not detect PfRON2 in the immunoprecipitant with anti-PfAMA1 Ab [7].

The association between the $83-\mathrm{kDa}$ proform of PfAMA1 with RON proteins raises the possibility that the processing of PfAMA1 from the $83-\mathrm{kDa}$ form to $66-\mathrm{kDa}$ form occurs not only in the microneme, as previously proposed [33], but also on the apical tip of the merozoite after release from the microneme in mature schizonts. If this is the case, it is not clear whether this AMA1 processing occurs after complex formation with RON proteins or is mainly achieved prior to this. However, it is formally possible that disruption of the different intracellular microorganelles during the experimental procedure resulted an artificial complex formation of PfAMA1 proform, for which further studies are required.

Due to the fact that $P$. falciparum AMA1 exhibits relatively high polymorphism between lines, which is considered to be generated by positive diversifying selection under the human immune pressure, we evaluated the polymorphic nature of PfRON2. Although the level of polymorphism of RON2 is not high, the fact that $d_{\mathrm{N}}>d_{\mathrm{S}}$ suggests that positive diversifying 
selection does indeed act on RON2. Three types of amino acid substitutions found at aa 1464 (Asp, Glu, and Gly) suggests that this particular site is under diversifying selection and is possibly to be exposed to host immunity. Thus, PfRON2 not only appears to have an important role in host cell invasion by apicomplexan parasites, but also is a potential target for malaria intervention strategies.

\section{Acknowledgements}

We thank N Iyoku for her expertise, I Ling for anti-PfRhopH2 serum, C Long for anti-PfAMA1 serum, anti-PfRON4 antibody (26C64F12) for J-F Dubremetz, and R Culleton for critical reading. Preliminary sequence data of P. knowlesi, P. berghei, P. chabaudi, and B. bigemina were produced by the corresponding groups at the Sanger Institute website at $\mathrm{http}: / / \mathrm{www}$.sanger.ac.uk/. Preliminary sequence data of $P$. vivax was produced at the Institute for Genomic Research website at http://www.tigr.org. This work was supported in part by Grants-in-Aid for Scientific Research 17590372 and 17406009 (to OK) from the Ministry of Education, Culture, Sports, Science and Technology, Japan. JC acknowledges the support of National Natural Science Foundation of China 30700695.

\section{References}

[1] Snow RW, Guerra CA, Noor AM, Myint HY, Hay SI. The global distribution of clinical episodes of Plasmodium falciparum malaria. Nature 2005;434:214-7.

[2] Kaneko O. Erythrocyte invasion: vocabulary and grammar of the Plasmodium rhoptry. Parasitol Int 2007;56:255-62.

[3] Aikawa M, Miller LH, Johnson J, Rabbege J. Erythrocyte entry by malarial parasites. A moving junction between erythrocyte and parasite. J Cell Biol 1978;77:72-82.

[4] Boothroyd JC, Dubremetz JF. Kiss and spit: the dual roles of Toxoplasma rhoptries. Nat Rev Microbiol 2008;6:79-88. 
[5] Alexander DL, Mital J, Ward GE, Bradley P, Boothroyd JC. Identification of the moving junction complex of Toxoplasma gondii: a collaboration between distinct secretory organelles. PLoS Pathog 2005;1:e17.

[6] Lebrun M, Michelin A, El Hajj H, Poncet J, Bradley PJ, Vial H, et al. The rhoptry neck protein RON4 re-localizes at the moving junction during Toxoplasma gondii invasion. Cell Microbiol 2005;7:1823-33.

[7] Alexander DL, Arastu-Kapur S, Dubremetz JF, Boothroyd JC. Plasmodium falciparum AMA1 binds a rhoptry neck protein homologous to TgRON4, a component of the moving junction in Toxoplasma gondii. Eukaryot Cell 2006;5:1169-73.

[8] Baum J, Tonkin CJ, Paul AS, Rug M, Smith BJ, Gould SB, et al. A malaria parasite formin regulates actin polymerization and localizes to the parasite-erythrocyte moving junction during invasion. Cell Host Microbe 2008;3:188-98.

[9] Triglia T, Healer J, Caruana SR, Hodder AN, Anders RF, Crabb BS, et al. Apical membrane antigen 1 plays a central role in erythrocyte invasion by Plasmodium species. Mol Microbiol. 2000;38:706-18.

[10] Hehl AB, Lekutis C, Grigg ME, Bradley PJ, Dubremetz JF, Ortega-Barria E, et al. Toxoplasma gondii homologue of Plasmodium apical membrane antigen 1 is involved in invasion of host cells. Infect Immun 2000;68:7078-86.

[11] Mital J, Meissner M, Soldati D, Ward GE. Conditional expression of Toxoplasma gondii apical membrane antigen-1 (TgAMA1) demonstrates that TgAMA1 plays a critical role in host cell invasion. Mol Biol Cell 2005;16:4341-9.

[12] Trager W, Jensen JB. Human malaria parasites in continuous culture. Science 1976;193:673-5. 
[13] Bahl A, Brunk B, Crabtree J, Fraunholz MJ, Gajria B, Grant GR, et al. PlasmoDB: the Plasmodium genome resource. A database integrating experimental and computational data. Nucleic Acids Res 2003;31:212-5.

[14] Tsuboi T, Takeo S, Iriko H, Jin L, Tsuchimochi M, Matsuda S, et al. The wheat germ cell-free based production of malaria proteins for discovery of novel vaccine candidates. Infect Immun 2008;76:1702-8.

[15] Ling IT, Kaneko O, Narum DL, Tsuboi T, Howell S, Taylor HM, et al. Characterisation of the rhoph2 gene of Plasmodium falciparum and Plasmodium yoelii. Mol Biochem Parasitol 2003;127:47-57.

[16] Kaneko O, Yim Lim BY, Iriko H, Ling IT, Otsuki H, Grainger M, et al. Apical expression of three RhopH1/Clag proteins as components of the Plasmodium falciparum RhopH complex. Mol Biochem Parasitol 2005;143:20-8.

[17] Kaneko O, Fidock DA, Schwartz OM, Miller LH. Disruption of the C-terminal region of EBA-175 in the Dd2/Nm clone of Plasmodium falciparum does not affect erythrocyte invasion. Mol Biochem Parasitol 2000;110:135-46.

[18] Torii M, Adams JH, Miller LH, Aikawa M. Release of merozoite dense granules during erythrocyte invasion by Plasmodium knowlesi. Infect Immun 1989;57:3230-3.

[19] Aikawa M, Atkinson CT. Immunoelectron microscopy of parasites. Adv Parasitol 1990;29:151-214.

[20] Bendtsen JD, Nielsen H, von Heijne G, Brunak S. Improved prediction of signal peptides: SignalP 3.0. J Mol Biol 2004:340;783-95.

[21] Hofmann K, Stoffel W. TMbase - A database of membrane spanning proteins segments. Biol Chem. Hoppe-Seyler 1993;374:166. 
[22] Krogh A, Larsson B, von Heijne G, Sonnhammer EL. Predicting transmembrane protein topology with a hidden Markov model: application to complete genomes. $\mathrm{J}$ Mol Biol 2001:305;567-80.

[23] Linding R, Russell RB, Neduva V, Gibson TJ. GlobPlot: Exploring protein sequences for globularity and disorder. Nucleic Acids Res 2003;31:3701-8.

[24] Edgar RC. MUSCLE: multiple sequence alignment with high accuracy and high throughput. Nucleic Acids Res 2004;32,1792-7.

[25] Tamura K, Dudley J, Nei M, Kumar S. MEGA4: Molecular Evolutionary Genetics Analysis (MEGA) software version 4.0. Mol Biol Evol 2007;24:1596-9.

[26] Altschul SF, Madden TL, Schäffer AA, Zhang J, Zhang Z, Miller W, et al. Gapped BLAST and PSI-BLAST: a new generation of protein database search programs. Nucleic Acids Res 1997;25:3389-3402.

[27] Pain A, Renauld H, Berriman M, Murphy L, Yeats CA, Weir W, et al. Genome of the host-cell transforming parasite Theileria annulata compared with T. parva. Science 2005;309:131-3.

[28] Gardner MJ, Bishop R, Shah T, de Villiers EP, Carlton JM, Hall N, et al. Genome sequence of Theileria parva, a bovine pathogen that transforms lymphocytes. Science 2005;309:134-7.

[29] Le Roch KG, Zhou Y, Blair PL, et al. Discovery of gene function by expression profiling of the malaria parasite life cycle. Science 2003;301:1503-8.

[30] Howell SA, Withers-Martinez C, Kocken CH, Thomas AW, Blackman MJ. Proteolytic processing and primary structure of Plasmodium falciparum apical membrane antigen-1. J Biol Chem 2001;276:31311-20. 
[31] Ghoneim A, Kaneko O, Tsuboi T, Torii M. The Plasmodium falciparum RhopH2 promoter and first 24 amino acids are sufficient to target proteins to the rhoptries. Parasitol Int 2007;56:31-43.

[32] Rungruang T, Kaneko O, Murakami Y, Tsuboi T, Hamamoto H, Akimitsu N, et al. Erythrocyte surface glycosylphosphatidyl inositol anchored receptor for the malaria parasite. Mol Biochem Parasitol 2005;140:13-21.

[33] Healer J, Triglia T, Hodder AN, Gemmill AW, Cowman AF. Functional analysis of Plasmodium falciparum apical membrane antigen 1 utilizing interspecies domains. Infect Immun 2005;73:2444-51.

\section{Figure legends}

Figure 1. Schematic representation of PfRON2. S and TM indicate putative signal peptide (aa $1-20$ ) and transmembrane sequences, respectively. The shaded box indicates an interspecies variable region. Vertical red bars indicate conserved Cys residues among orthologous sequences. Homologous region between RhopH1/Clag and RON2 is indicated by a yellow box. The region used to generate anti-PfRON2 sera $(\alpha-P f R O N 2)$ and the region sequenced in the laboratory lines (sequencing) are indicated. Asterisks indicate polymorphic sites.

Figure 2. Amino acid alignment of Plasmodium RON2 and RhopH1/Clag. Alignment was generated by MUSCLE [24] with manual correction. "*" indicates that the residues in that column are identical in all sequences in the alignment. ":" indicates conserved substitutions and "." indicates semi-conserved substitutions. In addition to 9 RON2 sequences, $P$. falciparum Clag2 (AAC71977), Clag9 (CAD52032), P. yoelii RhopH1A (BAB70675), 
RhopH1AP (BAB70677), and PvRhopH1 (contig 1047) were used to generate the alignment. Cys residues are highlighted in red. The region possessing homology between RhopH1/Clag and RON2 as identified by BLASTP is indicated by the bar under the alignment.

Figure 3. Transcriptional analysis by quantitative RT-PCR of pfrhoph2, pfron2, and pfama1 genes during blood stages of $P$. falciparum (HB3 line). $Y$-axis indicates copy number of each transcript detected per 1000 parasites. Similar results were observed in 3 independent experiments (data not shown).

Figure 4. Western blot analysis of antisera against native parasite proteins. (A) Schizont-enriched parasite extracts were stained by rabbit preimmune serum, (Rab preimmune), rabbit anti-PfRON2 (Rab $\alpha-P f R O N 2$ ), mouse anti-fRON2N (Mo $\alpha-P f R O N 2$ ), and Abs against GST (Mo $\alpha$-GST) or PfAMA1 (Mo $\alpha$-PfAMA1) under both reducing and non-reducing conditions. Both mouse and rabbit anti-PfRON2 sera detected a band slightly larger than $250 \mathrm{kDa}$. (B) Western blot of schizont-enriched parasite extracts from 3 different $P$. falciparum lines, Dd2, 3D7, and HB3 with mouse anti-fRON2N serum. Arrows indicate predicted $P f R O N 2$ bands.

Figure 5. PfRON2 is co-precipitated with PfRON4 and PfAMA1. Schizont-rich parasite Triton X-100 extracts (Pf Tx extract) were immunoprecipitated (IP) with rabbit sera against PfRhopH2 ( $\alpha$-PfRhopH2), PfRON2 ( $\alpha$-PfRON2), PfAMA1 ( $\alpha$-PfAMA1) or mouse monoclonal Ab against PfRON4 ( $\alpha-P f$ RON4), then stained against PfRON2, PfAMA1, $P f R O N 4$, or $P f R h o p H 2$. AMA $1_{83}$ is a proprotein form and $A M A 1_{66}$ is a processed form.

Figure 6. PfRON2 is expressed at the apical end of Plasmodium merozoites. 
Schizont-infected erythrocytes and merozoites were dual-labeled with antisera against PfRON2 and PfAMA1 (A), PfClag3.1 (B), or PfRON4 (C). Merged images are shown in the right panels. All segmented schizonts and merozoites are positive for PfRON2. Nuclei are counterstained with DAPI. Colocalization of PfRON2 with PfRON4 (rhoptry neck marker) was observed but neither colocalized with PfClag3.1 (rhoptry body marker) nor PfAMA1 (microneme marker). To eliminate the background staining, negative control sera were always used and images were assessed (data not shown).

\section{Figure 7. Rhoptry neck localization of PfRON2 by immunoelectron microscopy.} Longitudinally sectioned merozoites in schizont-infected erythrocytes were labeled with anti-PfRON2 serum followed by secondary Ab conjugated with gold particles. Gold particles were restricted to the narrow neck portion of the rhoptries (R). Two different images are shown (A and B). N indicates nucleus. Bars $=200 \mathrm{~nm}$. 
Figure 1.

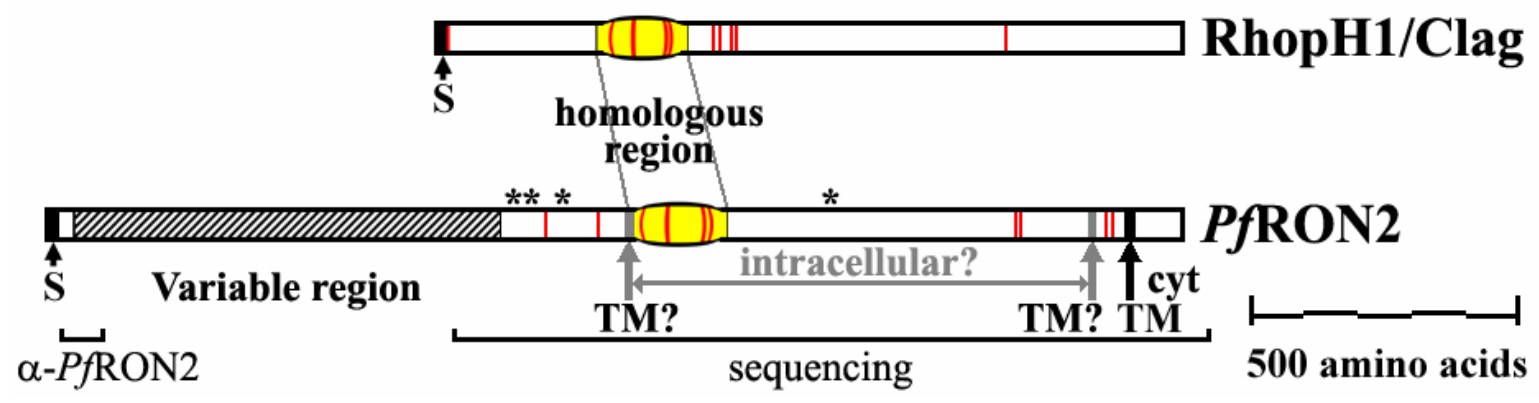




\section{Figure 2.}

\begin{tabular}{|c|c|c|c|c|c|}
\hline Protein & posit & Amino acid se & equence & & 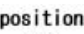 \\
\hline PyhopH1AP & 245 & YGIGIYNOIGSHYIALGHFITLKMAFKHFNKYFV-NGDLKFYTWGMILOFTPEDRFKALDLI & DD-TYPKKNKINKKNTIIKSRITGSSQD-- & WVLEFLIHOYNIY-OIEL & 351 \\
\hline PfClag9 & 245 & YHIGIFNMIGSHYIAVGHFITLKLALKNYKKYFE-IGSLKYLNWOSILKFNOSDRFKVLDLI & E-SSWYEGQMKRREOYLKNNIFSTSEE & SVLEFLIHHMNKY-OMEL & 351 \\
\hline PuRopH1 & 251 & GIAMYNIVGSNLVAL GHFVVLQLLALKKYDQFFK-FGKMRFFNWOKILSFSATDRFRVLD & IVQDVQ & NILEFLVHHFNKY--ONSL. & 361 \\
\hline PyRhopHIA & 233 & LSLALKNYENFFE-KGKGHF ISWOKLLSFNUSDRFKALD & & NILEFLIHYFNKY-QLSL & 343 \\
\hline PKRON2 & 1055 & SYNHYFV-QGLKHFYSLETMLMANSDY & & NAYTLI--NIHE & 1174 \\
\hline & & & & TLI-NVHE & 1234 \\
\hline & & & & NINE & 1246 \\
\hline & & & & -NINE & 1111 \\
\hline TaRON2 & 422 & LGADMFWVSGNNPFILGHLATMMLGYTEYESFFSDYPKRRFYSWLELVRSGPGGSINRLDYM & GSRYARC & NLTMDSMGD & 541 \\
\hline TPRON2 & 424 & LGADMFWVSGNNPFILGHLATMMLGYTEYESFFSDYPKRRFYSWLELVRSGPGGSVNRLDYM & & NLTMDSMGD & 543 \\
\hline BbigRON2 & 343 & LGMELFAACGNNPFLLGHLATNMLAYTQYKLFFDGDQGRPFYTWLDLVKSG--NLDMLDR & & & 459 \\
\hline & & $\therefore \quad \therefore \quad: * \quad:$ & $\cdots$ & . & \\
\hline
\end{tabular}

region identified by BLASTP search

PYRhopH1AP 352 FKKASILN--LNMOLFLDTDSLKONFFNFUIKKKNNE----DIYKGPHFKOE 397

PfClag9 352 YSKMHKLLS---LNVOIYLENKHLKEKFLOFM RSRKE--- NIYESDRFKOE 397

P.RhopHI 362 VSGLYQED-_-FKTHYLOEHSDIRNEFFKFN NDINH-_- NIYSSKLFLSE 403

PYRhopHIA 344 ISRTYGDE---FQSYYLLEHKDMKEEFFRFM DSSKN--- NIYNSSIFLSN 358

$P F C$ lag2 401 IKATQDTD-FELHGMMEHKNIKDYFFSFN NDPKE- 11 -

PKRON2 1175 IOKVFNNSDTYGYENSISFAHNAVR-IFSOV PRDDAKNT-LS DFEKSTLYNPK 1223

$\begin{array}{lll}\text { PURON2 } & 1235 & \text { IOKVFNISEAYGYENSISFGHNAVR--IFSOV PRDDAKNT-FG DFEKSTLYNSK } \\ 1283\end{array}$

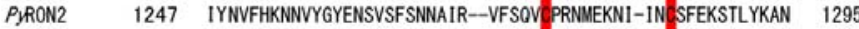

PBRON2 1112 VYNVFHIKNVYYGYENSVSFSNNAIR-YFSOV PRNMEKNI-IN SFEKSTLYKGN 1160

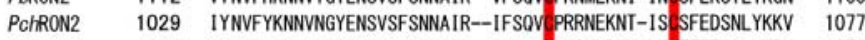

PARON2 1225 INNVFSNNNVDGYENSLSFSHNAIR-IFSKV PKINNDNV-LK EFEESSLYNPK 1273

IaRON2 542 LLNOKGGN--YEPNVGSI INGKR--MOAKL SNPGDGTIAVR DFEHSRLLGEE 591

IDRON2 544 LLNQKGGS--YYPNVGSI INGKR--MQAKL SNPGDGTIAVR DFEHSVLVGEE 593

BbigRON2 460 LLSQYGVP--YYHSVGAIANGRR-MOAFV SDPRESAIVVRCDFMSSTLNSSK 509 
Figure 3.

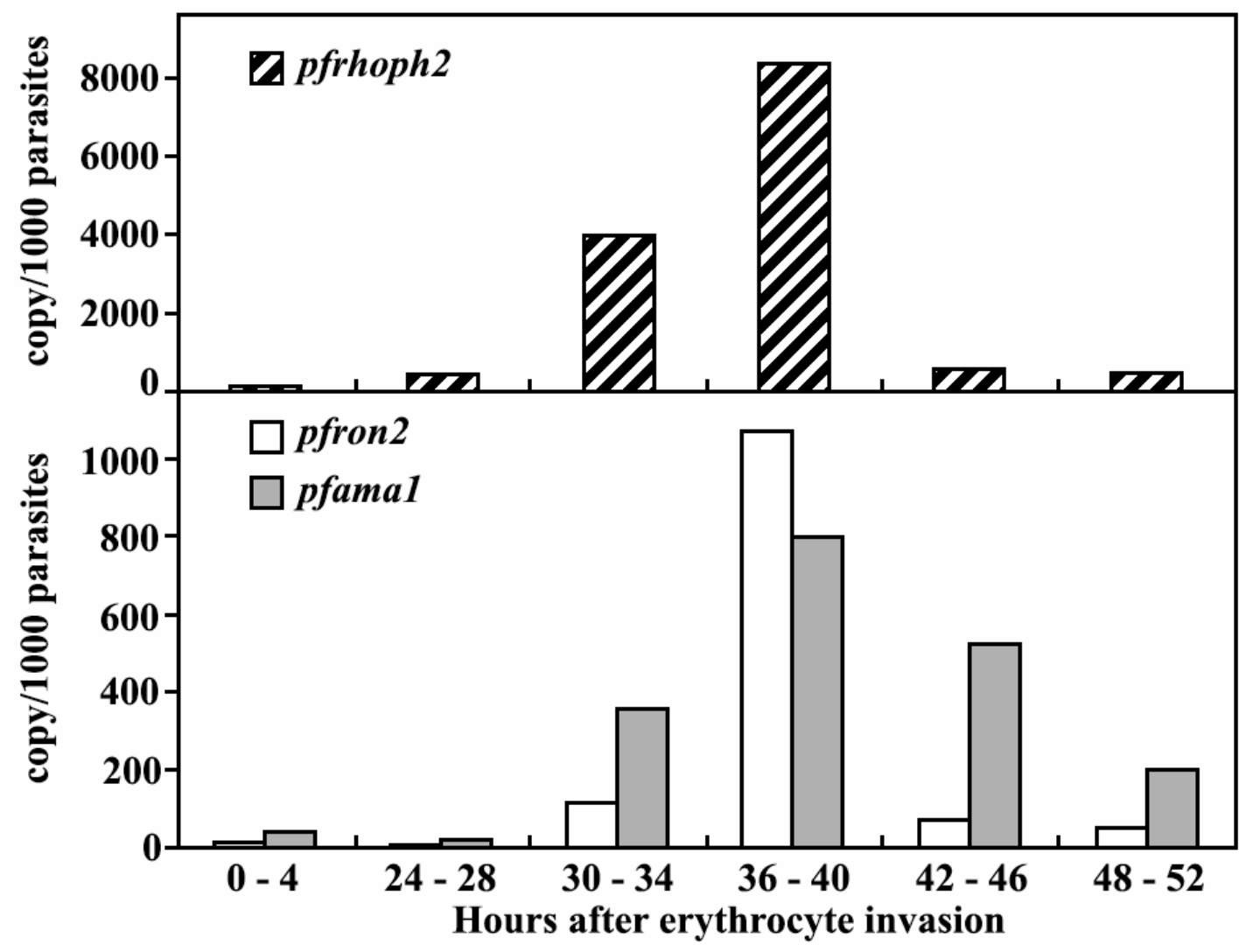


Figure 4.

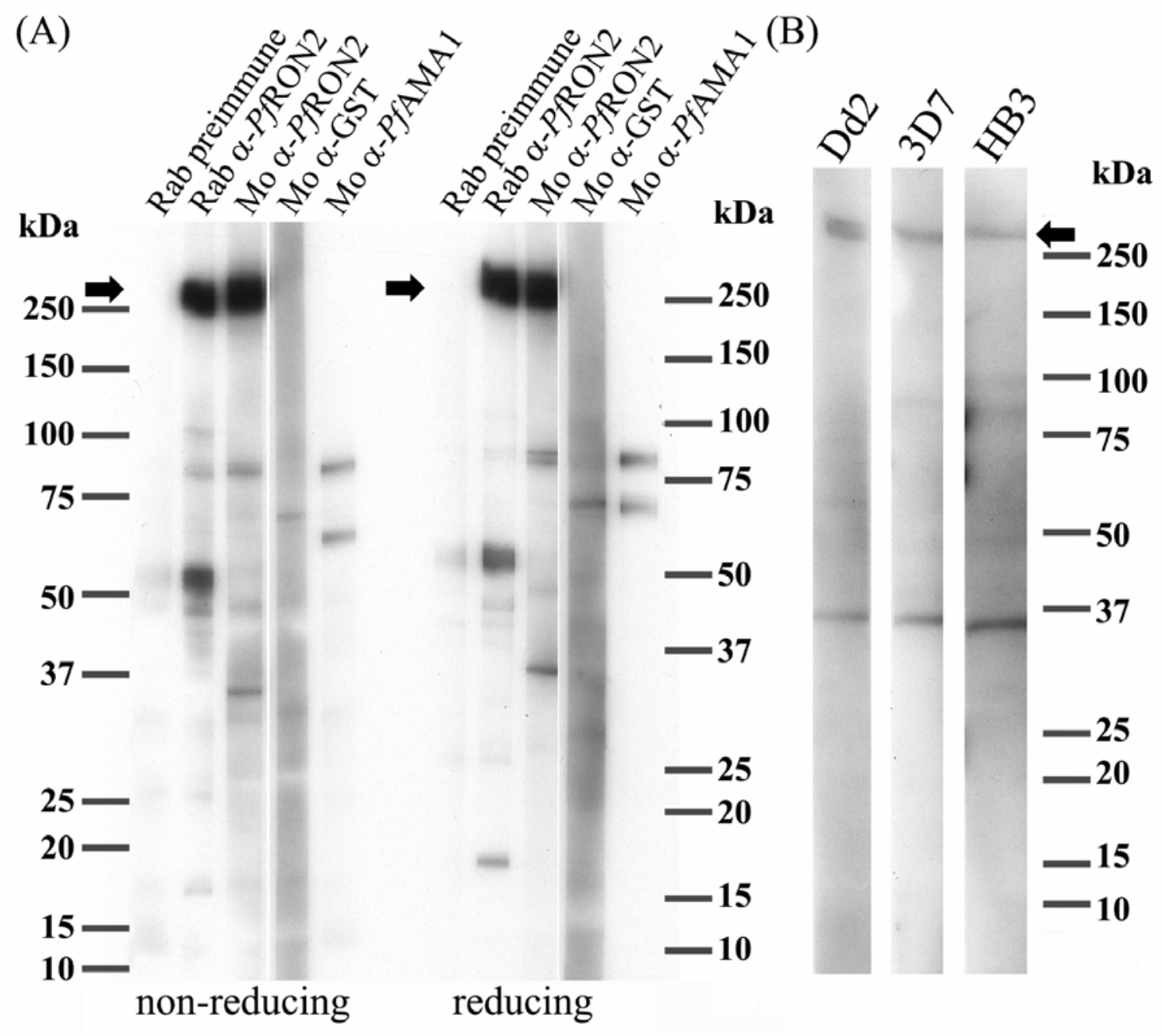


Figure 5.

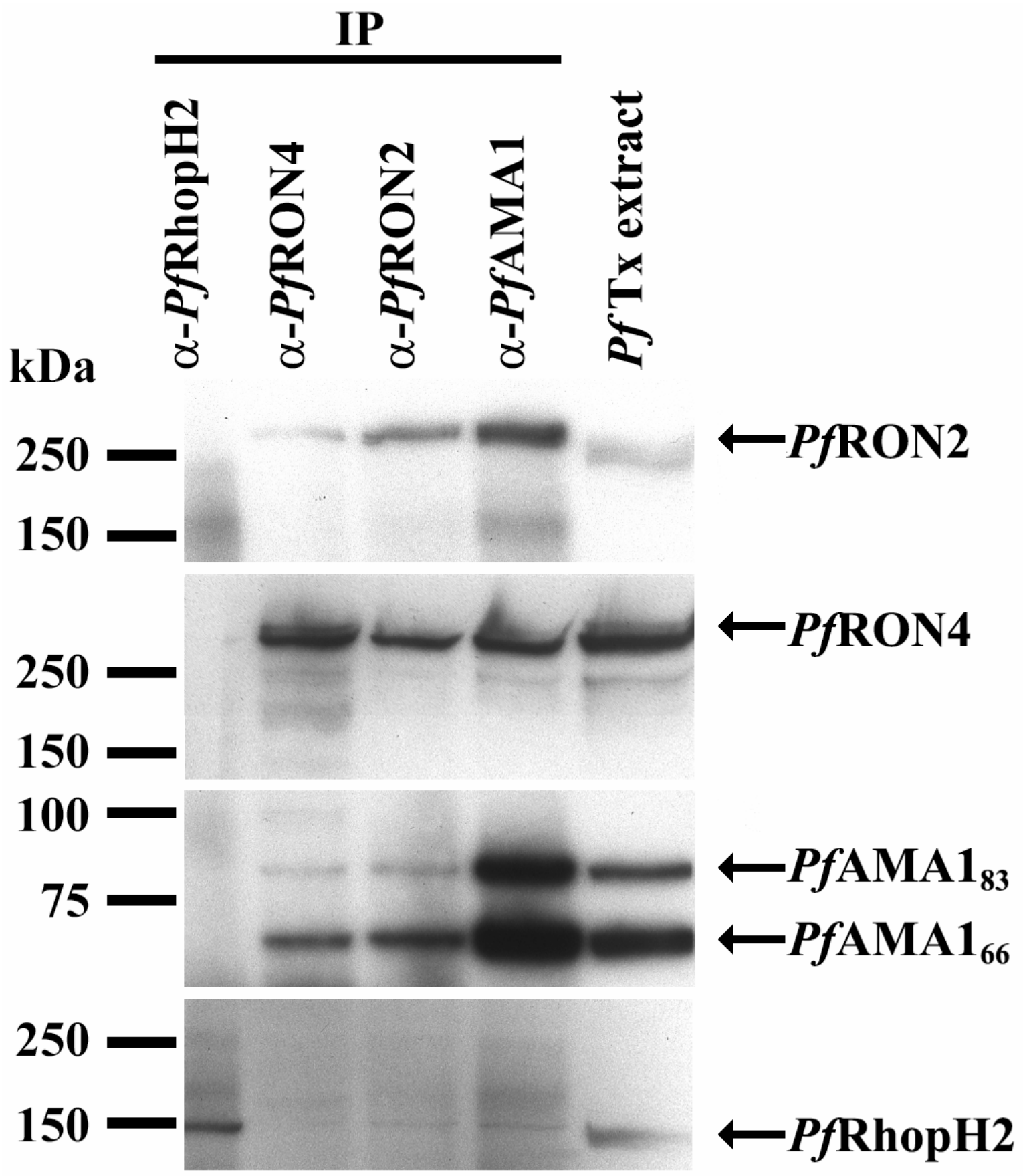


Figure 6.

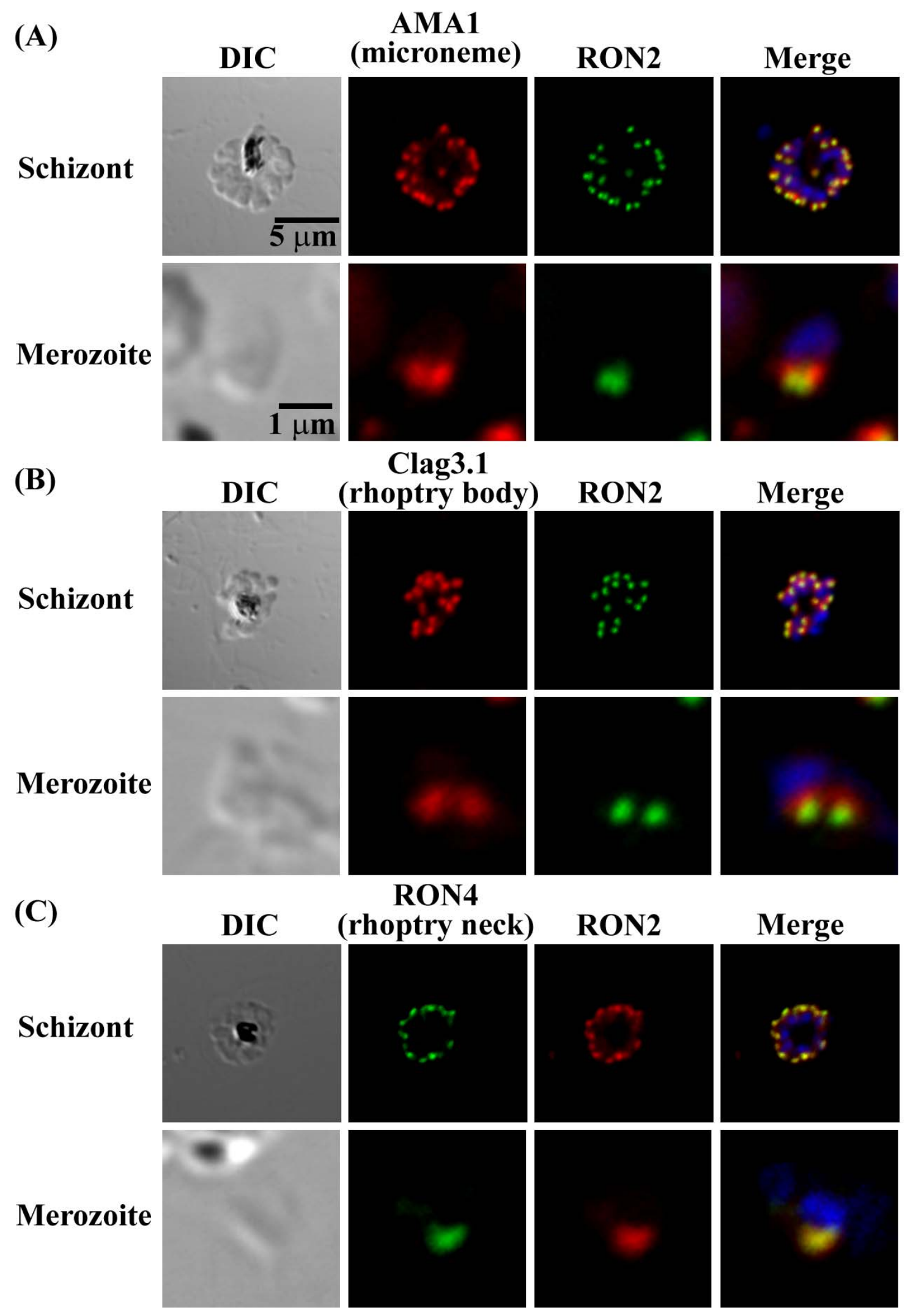


Cao et al. - 30 -

Figure 7.

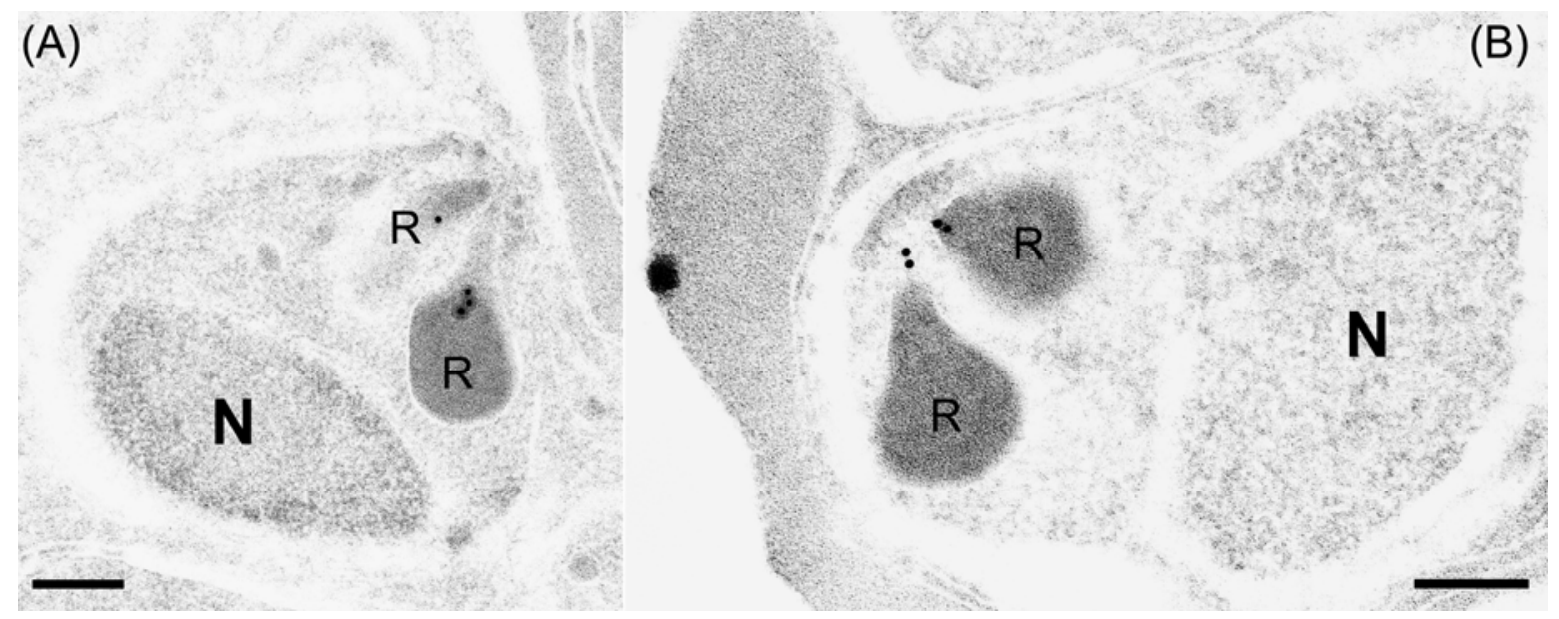

